\title{
Use of ambient lighting during colonoscopy and its effect on adenoma detection rate and eye fatigue: results of a pilot study
}

\section{(ㄷ)(i) $\Theta$}

Authors

Ryan T. Hoff ${ }^{1}$, Andrew Mazulis' ${ }^{1}$, Meghana Doniparthi ${ }^{1}$, Assad Munis ${ }^{1}$, Anne Rivelli², Asif Lakha ${ }^{1}$, Eli Ehrenpreis ${ }^{1,3}$

Institutions

1 Advocate Lutheran General Hospital - Medicine, Park Ridge, Illinois, United States

2 Advocate Lutheran General Hospital - Russell Research Institute, Park Ridge, Illinois, United States

3 Rosalind Franklin University of Medicine and Science Chicago Medical School - Medicine, North Chicago, Illinois, United States

submitted 29.9.2020

accepted after revision 20.1.2021

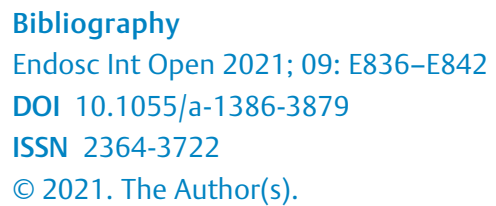

This is an open access article published by Thieme under the terms of the Creative Commons Attribution-NonDerivative-NonCommercial License, permitting copying and reproduction so long as the original work is given appropriate credit. Contents may not be used for commercial purposes, or adapted, remixed, transformed or built upon. (https://creativecommons.org/licenses/by-nc-nd/4.0/)

Georg Thieme Verlag KG, Rüdigerstraße 14,

70469 Stuttgart, Germany

Corresponding author

Ryan T. Hoff, Advocate Lutheran General Hospital - Medicine, 1775 Dempster Street, Park Ridge, IL 60068, United States Fax: +1-847-696-3391

ryan.hoff@aah.org

\section{ABSTRACT}

Background and study aims Adenoma detection rate (ADR) appears to decrease as the number of consecutive hours performing procedures increases, and eye strain may be a contributing factor. Ambient light may improve symptoms of eye strain, but its effects have yet to be explored in the field of gastroenterology. We aim to determine if using ambient lighting during screening colonoscopy will maintain ADRs and improve eye strain symptoms compared with low lighting.

Methods At a single center, retrospective data were collected on colonoscopies performed under low lighting and compared to prospective data collected on colonoscopies with ambient lighting. Eye fatigue surveys were completed by gastroenterologists. Satisfaction surveys were completed by physicians and staff.

Results Of 498 low light and 611 ambient light cases, 172 and 220 adenomas were detected, respectively $(P=0.611)$. Under low lighting, the ADR decreased $5.6 \%$ from first to last case of the day $(P=0.2658)$. With ambient lighting, the ADR increased by $2.80 \%(P=0.5445)$. The difference in the overall change in ADR between first and last cases with ambient light versus low light was statistically significant $(8.40 \%$ total unit change, $P=0.01)$. The average eye strain scores were 8.12 with low light, and 5.63 with ambient light $(P=0.3341)$.

Conclusions Performing screening colonoscopies with ambient light may improve the differential change in ADR that occurs from the beginning to the end of the day. This improvement in ADR may be related to improvement in operator fatigue. The effect of ambient light on eye strain is unclear. Further investigation is warranted on the impact of ambient light on symptoms of eye strain and ADR.

\section{Introduction}

Colorectal cancer (CRC) ranks 3 rd in cancer-related mortality for men and women, with an estimated 149,500 new cases and 52,980 deaths in the United States in 2021 [1]. From 1969 to 2017 , colorectal cancer mortality has decreased by more than $50 \%$,[1] with a particularly steep decline of $3 \%$ per year in the early 2000 s [2]. The incidence and mortality of colorectal cancer is declining, which is associated with improvements in colon cancer screening rates and quality of colonoscopy. These improvements include technological advances, improved bowel preparation, and the application of quality parameters, such 
as adenoma detection rate (ADR). Data from the Nurses' Health Study and the Health Professionals Follow-up cohort demonstrate colonoscopy is associated with a reduced incidence of both distal and proximal CRC [3].

CRC screening programs are cost-effective, but require significant resources [4], and efforts to improve the performance of colonoscopy as a screening tool have been the focus of a substantial and growing body of research. Payers are shifting towards value-based reimbursement, and hospital systems as well as physicians are looking at ways to provide high-value care. Colonoscopy-related quality measures have been identified to establish standards in the performance of colonoscopies, with ADR standing out as the most important quality metric [5]. An increase in adenoma detection is associated with a lower risk of colorectal cancer mortality [6]. Identifying factors that increase ADR are currently being investigated with the aim of optimizing colonoscopy effectiveness. Two elements suspected of influencing ADR include endoscopist fatigue [7-9], procedure time, and the number of consecutive hours that colonoscopy is performed [10-13]. Almadi et al found ADR decreases if colonoscopy is performed $\leq 3$ hours compared to $>3$ hours after the start of each endoscopy session [7]. Performing procedures in half-day blocks appears to improve ADR, especially for afternoon procedures [14]. While some studies have not shown procedure time to impact ADR $[15,16]$, this may be partially due to a variable effect of timing of day and fatigue on individual physicians [9]. The role of eye fatigue, also known as "computer vision syndrome", has received attention in radiology literature $[17,18]$, but has heretofore not been addressed as it relates to the performance of colonoscopy.

The term "computer vision syndrome," also known as digital eye strain, describes eye symptoms in persons exposed to computer screens and video display units (VDU) with flat-panel displays. In 2016, Porcar et al. evaluated the presence of eye symptoms in amongst one hundred sixteen non-presbyopic VDU users with flat-panel displays [19]. Seventy-two percent of participants reported eye symptoms related to VDU use [19]. Eye symptoms increased with duration of VDU use, and markedly increased with more than six hours of screen use $(P=$ $0.01)$. Computer use has increased in healthcare with the growing use of electronic medical records; healthcare workers may experience a high prevalence of computer vision syndrome, with symptoms corresponding to increased screen time [20].

The relationship between eye strain and ambient light has been explored in the field of radiology, and several studies have investigated optimal lighting conditions for the interpretation of dental imaging [21-25]. Evidence suggests that eye strain symptoms are prevalent amongst radiologists [26], and excessively bright lighting may negatively affect diagnostic accuracy [27]. Krupinski et al. found that radiologists experience more symptoms of eye strain at the end of a work day, and diagnostic accuracy is worse at the end of the work day [18]. Differences in adaptation between a bright screen and a room with low lighting may contribute to eye strain [28]. Typical radiology reading rooms have ambient light of 1 to 60 lux. For most liquid crystal display (LCD) monitors with standard settings, ambient light may be increased to 75 to 150 lux, which may improve symptoms of eye strain [28].

The above studies have found that the number of hours a physician performs procedures consecutively affects ADR, and that the more hours spent using a VDU, the more eye symptoms people experienced. There are limited data available regarding eye symptoms related to flat-panel displays. Adaptation between a bright screen and a room with low lighting and its contribution to eye strain as a day progresses is yet to be explored in the field of gastroenterology.

The aims of this study were to determine if use of ambient lighting vs low lighting during screening colonoscopy affects adenoma detection rates, to determine if ambient lighting is associated with fewer symptoms of strain in colonoscopists, and to explore physician preferences regarding lighting conditions.

\section{Methods}

We conducted a single-center study at an independent community-based teaching hospital comparing ADR in screening colonoscopies performed in low lighting with those performed with ambient lighting. Low lighting was defined as $<75$ lux, whereas ambient lighting was defined as 75 to 150 lux. All cases included in the study involved adult patients undergoing screening colonoscopy with a participating gastroenterologist. Diagnostic colonoscopies, history of colon resection, colorectal cancer, and cases performed in children, pregnant women, and prisoners were excluded from analysis. Cases involving gastroenterology fellows were also excluded.

Eight male gastroenterologists and one female gastroenterologist participated in the study. A tenth gastroenterologist expressed intent to participate, but left their practice, and was thus removed from the study. The average age of participating gastroenterologists was 48 years. Each physician served as their own control. Seven of the nine participating physicians required corrective lenses. Physicians requiring visual correction used updated corrective lenses during all procedures. For the retrospective data collection portion of the study, participating gastroenterologists performed their entire day of procedures with low lighting, including diagnostic procedures. Use of narrowband imaging was to the discretion of the physician. All procedures were performed with high-definition Olympus colonoscopes (EVIS EXERA III PCF-H190L and EVIS EXERA III CFHQ190 L). All monitors used for endoscopy were 42-inch highdefinition screens; the same monitors were used in both the retrospective and prospective arms.

Retrospective data were collected over a 6-month period on screening colonoscopies performed in procedure rooms with low lighting from January 2017 to June 2017. Low lighting was defined as lux $<75$. Data included any adenoma detection during screening colonoscopy. Adenoma detection rates were calculated, and defined by the percentage of screening colonoscopies where at least one adenomatous polyp was found. Withdrawal time was measured, which was defined by cecum time to completion of colonoscopy. Overhead lights were turned off for these procedures, leaving only minimal light from equip- 
- Table 1 Symptom assessment survey.

\begin{tabular}{|c|c|c|c|c|c|c|c|}
\hline & None & Slight & Mild & Moderate & Somewhat bad & Bad & Severe \\
\hline $\begin{array}{l}\text { Blurred vision at near distances, e. g. book or newspaper } \\
\text { (with your usual glasses or contact lenses). }\end{array}$ & 0 & 1 & 2 & 3 & 4 & 5 & 6 \\
\hline $\begin{array}{l}\text { Blurred vision at intermediate distances, e. g. comput- } \\
\text { er screen (with your usual glasses or contact lenses) }\end{array}$ & 0 & 1 & 2 & 3 & 4 & 5 & 6 \\
\hline $\begin{array}{l}\text { Blurred vision at far distances, e. g. computer screen } \\
\text { (with your usual glasses or contact lenses) }\end{array}$ & 0 & 1 & 2 & 3 & 4 & 5 & 6 \\
\hline $\begin{array}{l}\text { Difficulty or slowness in refocusing my eyes from one } \\
\text { distance to another }\end{array}$ & 0 & 1 & 2 & 3 & 4 & 5 & 6 \\
\hline Irritated or burning eyes & 0 & 1 & 2 & 3 & 4 & 5 & 6 \\
\hline Dry eyes & 0 & 1 & 2 & 3 & 4 & 5 & 6 \\
\hline Eyestrain & 0 & 1 & 2 & 3 & 4 & 5 & 6 \\
\hline Headache & 0 & 1 & 2 & 3 & 4 & 5 & 6 \\
\hline Tired eyes & 0 & 1 & 2 & 3 & 4 & 5 & 6 \\
\hline Sensitivity to bright lights & 0 & 1 & 2 & 3 & 4 & 5 & 6 \\
\hline
\end{tabular}

ment in the room (low light). At the start of the study, measurements with a lux meter confirmed low lighting in the range of 0 to 75 lux.

Prospective data were then collected on colonoscopies performed with ambient lighting during the same 6 calendar months the following year, from January 2018 to June 2018. Clinical engineering adjusted room lighting to 75 to 150 lux for the prospective cases. Measurements with a lux meter confirmed ambient lighting in the range of 75 to 150 lux.

The first case of the day was defined as the first screening colonoscopy performed during the day of procedures. If only one screening colonoscopy was performed, then this was categorized as a "first case" if performed during the first half of the day of procedures or as the "last case" if performed during the latter half of the day of procedures.

A validated eye fatigue survey was completed by all gastroenterologists before and after the use of ambient lighting ( $\downarrow$ Table 1 ), with the extent of symptoms rated on a scale of 0 to 6 (adapted from Hayes, et al) [29]. An eye strain score was calculated as the sum of the numerical responses to each question for each participant. The lowest possible eye strain score was 0 , and the highest possible score was 60 . Eye strain scores were compared before and after the use of ambient light. Satisfaction surveys were completed by gastroenterologists, anesthesiologists, nurses, and technicians ( $\triangleright$ Table 2 and $\triangleright$ Table 3 ).

A power calculation was performed using GraphPad StatMate 2 software (GraphPad Software Inc, San Diego CA). With an expected ADR of $35 \%$ and $80 \%$ power, 400 subjects in each group were necessary to detect a $10 \%$ improvement in ADR. Summary statistics were calculated for self-report symptom assessment and satisfaction surveys ( ADR was calculated for each physician separately by lighting conditions (i.e. low lighting vs. ambient lights) and averaged for overall ADR by lighting conditions ( $>$ Table 4). Pearson Chisquare tests were performed to compare differences in ADR, in- cluding change from first to last cases between lighting conditions ( $\triangleright$ Table 5 ). Comparison of eye strain score in low light and ambient light was performed using Wilcoxon matchedpairs signed-ranks test. Analyses were performed using SAS, version 9.4 (SAS Institute, Cary North Carolina, United States) and InStat (GraphPad Software, San Diego, California, United States). This study was approved by the Advocate Institutional Review Board (IRB \# 6665). This study was registered with clinicaltrials.gov (NCT04441242).

\section{Results}

We compared 498 retrospectively collected control cases performed with low lighting with 611 prospectively collected intervention cases performed with ambient lighting by the same nine gastroenterologists. Of the 498 low light cases, 172 adenomas were detected, with an ADR of $34.5 \%$. Of the 611 ambient light cases, 220 adenomas were detected, with an ADR of $36.0 \%$ $(P=0.6109)$. Of the cases screened with low lighting, the ADR was $37.5 \%$ among the first cases of the day and $31.9 \%$ among last cases of the day, demonstrating a $5.6 \%$ decrease in ADR $(P=0.2658)$. Of the cases screened with ambient lighting, the ADR was $34.1 \%$ among first cases of the day and $36.9 \%$ among last cases of the day, representing a $2.80 \%$ increase $(P=0.5445)$. The difference in the overall difference in ADR between first and last cases when screened with ambient lighting $(+2.80 \%)$ versus low lighting $(-5.60 \%)$, was statistically significant (8.40\% total unit change, $P=0.01$, $>$ Table 3 and $>$ Table 4 ). Average withdrawal times were 11.8 minutes in the retrospective group and 11.8 minutes in the prospective group. In the retrospective arm, $51.0 \%$ of individuals undergoing screening colonoscopy were male and $49.0 \%$ were female, with an average age of 58.9 years. In the prospective arm, $42.7 \%$ were male and $57.3 \%$ were female, with an average age of 58.9 years. A logistical regression analysis was performed. After controlling for sex, age and pro- 
- Table2 Ambient Light Satisfaction Survey results from staff (nurses, technicians and anesthesiologists).

\begin{tabular}{|c|c|c|c|c|c|}
\hline & Strongly disagree & Disagree & Neutral & Agree & Strongly agree \\
\hline I prefer working with the lights on & $13.6 \%$ & $22.7 \%$ & $27.3 \%$ & $22.7 \%$ & $13.6 \%$ \\
\hline Completing my work is easier & $0 \%$ & $31.8 \%$ & $36.4 \%$ & $18.2 \%$ & $13.6 \%$ \\
\hline It is easier to detect changes in patient's clinical status & $4.5 \%$ & $18.1 \%$ & $31.8 \%$ & $31.8 \%$ & $13.6 \%$ \\
\hline $\begin{array}{l}\text { Communication between members of the healthcare team } \\
\text { improve with the lights on }\end{array}$ & $4.5 \%$ & $27.3 \%$ & $45.4 \%$ & $13.6 \%$ & $9.1 \%$ \\
\hline I experience fewer symptoms of eye strain with the lights on & $18.1 \%$ & $4.5 \%$ & $40.9 \%$ & $22.7 \%$ & $13.6 \%$ \\
\hline
\end{tabular}

- Table 3 Ambient Light Satisfaction Survey results from gastroenterologists.

\begin{tabular}{|c|c|c|c|c|c|}
\hline & Strongly disagree & Disagree & Neutral & Agree & Strongly agree \\
\hline I prefer working with the lights on & $0 \%$ & $33 \%$ & $22 \%$ & $11 \%$ & $33 \%$ \\
\hline Completing my work is easier & $0 \%$ & $22 \%$ & $33 \%$ & $22 \%$ & $22 \%$ \\
\hline It is easier to detect changes in patient's clinical status & $0 \%$ & $11 \%$ & $11 \%$ & $44 \%$ & $33 \%$ \\
\hline $\begin{array}{l}\text { Communication between members of the healthcare team } \\
\text { improve with the lights on }\end{array}$ & $0 \%$ & $11 \%$ & $44 \%$ & $22 \%$ & $33 \%$ \\
\hline I experience fewer symptoms of eye strain with the lights on & $0 \%$ & $0 \%$ & $44 \%$ & $22 \%$ & $33 \%$ \\
\hline
\end{tabular}

cedure time, the effect of ambient lighting on adenoma detection increased, approaching statistical significance $(O R=1.281$ $[0.981,1.674] ; P=0.0689)$.

Results of eye strain scores before and after ambient light are shown in $>$ Fig. $\mathbf{1}$, listed by physician. The average eye strain score prior to ambient light was 8.12, compared with 5.63 after ambient light $(P=0.3341)$. Two physicians who previously described symptoms did not experience any symptoms after using ambient light. Eye Strain Score with Low Light vs Ambient Light.

Results of satisfaction surveys for all staff are shown in $>$ Table 2 (gastroenterologists, nurses, technicians, anesthesiology). Of the respondents, $64.6 \%$ agreed, strongly agreed or were neutral regarding preference working with ambient light. Some $19.4 \%$ strongly agreed they preferred working with ambient light, compared with $9.7 \%$ who strongly disagreed. Of the respondents, $71 \%$ agreed, strongly agreed or were neutral that working with ambient lighting made completion of their work easier. Of the respondents, 54\% felt ambient light made it easier to detect changes in a patient's status. Of the respondents, $16.1 \%$ strongly agreed that communication between members of the healthcare team improved with ambient light, compared with $3.2 \%$ who strongly disagreed. Of respondents, $80.7 \%$ agreed, strongly agreed or were neutral that they experienced fewer symptoms of eye strain working with ambient light.

Results of satisfaction surveys of gastroenterologists are shown in $>$ Table 3 . The majority of gastroenterologists agreed or strongly agreed that it was easier to detect changes in patient's clinical status (77\%) and communication between members of the healthcare team was improved with the use of ambient light (55\%). The majority of gastroenterologists (55\%)

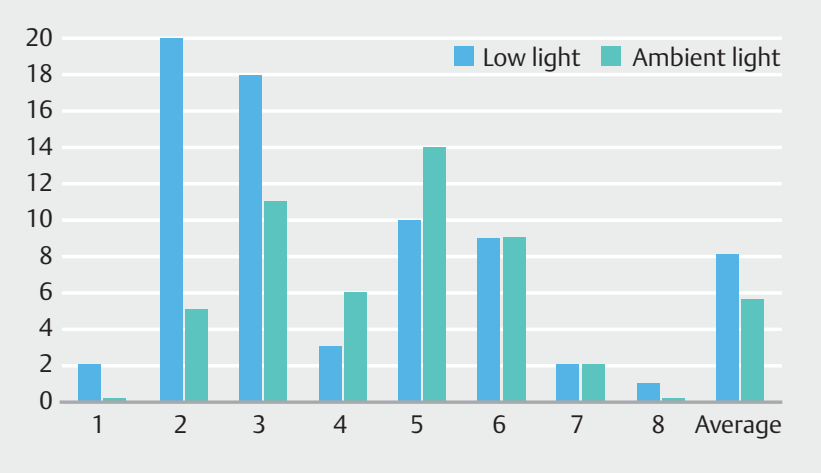

- Fig. 1 Eye strain scores reported by physicians (numbered 1-8) before ambient light (first bar columns, shown in blue) and after ambient light (second bar column, shown in green). Physicians 1 and 8 reported no symptoms after ambient light. Data is shown for all physicians with complete eye strain survey data (one physician in the study had incomplete eye strain data). The average eye strain scores are indicated by the final columns, with an average eye strain score of 8.12 after low light and 5.63 after ambient light.

agreed or strongly agreed that they experienced fewer symptoms of eye strain with ambient light. The majority of gastroenterologists agreed, strongly agreed, or were neutral that they preferred working with ambient light (66\%) and completing their work was easier (77\%). 
- Table4 Screening colonoscopy ADR for participating physicians with low lighting and ambient lighting.

\begin{tabular}{|l|c|c|c|c|}
\hline & All cases screened with low lighting & & All cases screened with ambient lighting \\
\hline Participant & Total screening colonoscopies & Overall ADR & Total screening colonoscopies & Overall ADR \\
\hline Physician 1 & 4 & $50.0 \%$ & 14 & $50.0 \%$ \\
\hline Physician 2 & 103 & $29.1 \%$ & 125 & $22.4 \%$ \\
\hline Physician 3 & 54 & $48.1 \%$ & 38 & $50.0 \%$ \\
\hline Physician 4 & 75 & $30.7 \%$ & 142 & $43.7 \%$ \\
\hline Physician 5 & 65 & $33.8 \%$ & 85 & $40.0 \%$ \\
\hline Physician 6 & 18 & $44.4 \%$ & 5 & $20.0 \%$ \\
\hline Physician 7 & 4 & $50.0 \%$ & 1 & $0.0 \%$ \\
\hline Physician 8 & 61 & $47.5 \%$ & 68 & $51.5 \%$ \\
\hline Physician 9 & 114 & $26.3 \%$ & 133 & $25.6 \%$ \\
\hline Overall & 498 & $34.5 \%$ & 611 & $36.0 \%$ \\
\hline The overall difference in ADR (1.5\%) was not statistically significant $(P=0.611)$. & & \\
\hline
\end{tabular}

- Table 5 Change in ADR from first to last case with ambient lighting and low light conditions.

\begin{tabular}{|c|c|c|c|c|c|}
\hline & & Colonoscopies & ADR & \% difference & $P$ value \\
\hline \multirow[t]{2}{*}{ Low lighting ( $N=498)$} & First case & 200 & $37.5 \%$ & \multirow[t]{2}{*}{$-5.60 \%$} & \multirow[t]{4}{*}{0.0104} \\
\hline & Last case & 166 & $31.9 \%$ & & \\
\hline \multirow[t]{2}{*}{ Ambient lighting $(\mathrm{N}=611)$} & First case & 232 & $34.1 \%$ & \multirow[t]{2}{*}{$+2.80 \%$} & \\
\hline & Last case & 187 & $36.9 \%$ & & \\
\hline
\end{tabular}

\section{Discussion}

This study is the first to examine optimal lighting settings in the endoscopy suite. Screening colonoscopies are often performed with low light. Low light conditions during the performance of endoscopy could plausibly exacerbate the symptoms of eye strain. Eye strain tends to worsen as the day progresses, with the use of display monitors in rooms with low lighting. Although the $1.5 \%$ difference in overall ADR between all cases conducted with ambient lighting compared with low lighting was not statistically significant, this is an expected finding and is likely due to the minimal effect of eye strain on adenoma detection for cases performed early in the day. From the first case to the last case of the day, ADR increased by $2.8 \%$ in the ambient light group and decreased $5.6 \%$ in the low light group. The difference between these changes was statistically significant $(P=0.01)$. Our data suggest that performing screening colonoscopies with ambient light may improve the change in ADR from the beginning of the day to the end of the work day, as compared with low lighting, where ours and other studies have demonstrated that ADR decreases during a day of procedures [7].

Improvement in ADR between the first and last colonoscopy (as opposed to the expected decrease in ADR) may be related to improved symptoms of eye strain, even though the measured decrease in the average eye strain score (8.12 vs 5.63 ) did not reach statistical significance $(P=0.3341)$. This discrepancy may be due to inadequate sampling, as symptoms were assessed at only two points in time in a small group of endoscopists. Symptom recall may also have been affected by survey timing. For example, physicians completing the eye strain survey at the start of the day or work week may recall fewer symptoms when compared with physicians completing surveys at the end of the day. Of note, the overall burden of eye strain symptoms within this group of physicians was relatively low, so the detection of significant changes in eye strain in our group may not have been feasible. It is possible that ambient lighting could improve symptoms in populations with greater burden of eye strain. Female physicians may experience more symptoms of eye strain [17]. The distribution of gender within this study is similar to that observed with gastroenterologists nationwide; [30] as such, it is difficult to determine if there are gender differences in eye strain symptoms. Future studies could explore eye strain symptoms in the endoscopy suite with optimization of survey timing and frequency with additional focus on gender differences and groups with a higher prevalence of symptoms.

Additional means of objectively measuring eye strain include critical flicker-fusion (CCF) frequency and monitoring of blinking and squinting [31]. There is evidence supporting the use of CFF as a metric representing eye fatigue, with declining values correlating with symptoms of eye strain. Reduced blinking rate and incomplete blinking may also correspond to eye strain symptoms. These objective measures are used in conjunction 
with subjective measures of eye strain symptoms [31]. In our pilot study, we chose to focus directly on the symptoms of eye strain, as we believe this outcome has the most relevance to practicing physicians. Future studies may be considered to evaluate objective measures of eye strain, and their potential role in explaining differences in eye strain and variation in adenoma detection rate.

This study has a few limitations. First, by definition, this study is not randomized. The durability of lighting settings' effect on eye strain symptoms is not known. As such, we chose to evaluate eye strain after prolonged periods of time with consistent light settings. We chose to conduct the study during the same part of the year to avoid seasonal variation in environmental light exposure. The retrospective nature of the low light group precludes observer bias, whereas physician participants in the prospective group were aware of their involvement in a research study. In addition, the brightness of the light in endoscopy rooms cannot be directly measured in the retrospective group. However, the conditions, lighting settings, and equipment in the room were unchanged and post-hoc measurements of light intensity indicate the lighting retrospective group was within the low light range (<75 lux).

Our sample consisted of a small group of physicians, and the groups were independent in their exposure. Including the same physicians in both the low light and ambient light groups served to neutralize many confounding factors, such as years of experience, skill, education, etc.; while this limits generalizability, it improves the quality of such an analysis. The prospective group contained fewer males and more females than the retrospective group, which may lead to a lower prevalence of adenomas in the prospective population. This could mask the effect of ambient lighting on ADR. After controlling for gender, age and procedure time in a logistical analysis, the effect of ambient light on overall ADR approached significance. Some gastroenterologists in the study performed relatively few colonoscopies, and ADR varied by physician. We chose to include all participating physicians, because the impact of ambient light on different gastroenterologists may vary, and including data from more physicians may provide a more accurate understanding of the overall effect of lighting conditions on screening colonoscopy. We planned to look for correlates related to ADR in the future. However, our data consist of too small of a group of individuals for this type of analysis.

Finally, the use of narrow band imaging was allowed at the discretion of the physician performing colonoscopy. A meta-analysis published in 2019 suggests that the use of second-generation narrow band imaging during screening colonoscopy is associated with a higher ADR than high-definition white light endoscopy. We believe these limitations have minimal impacts on the results; however, randomized prospective trials would be useful in confirming our findings.

Satisfaction survey responses by physicians, nurses, and technicians appeared generally favorable for the use of ambient lighting, though this study was not designed to evaluate staff satisfaction. More respondents felt they experienced fewer symptoms of eye strain with ambient light (41\% vs 19\%), and most respondents felt ambient lighting made it easier to detect changes in patient status (54\%). While it is plausible that ambient lighting may lead to more timely recognition of changes in patient status, this study does not provide data to answer that question. Since completion of the study, three of the nine participating gastroenterologists have changed their practice and now routinely use ambient lighting during endoscopic procedures. Adjusting light settings to ambient light in the endoscopy suite may have a positive impact on satisfaction among physicians and staff.

\section{Conclusions}

In summary, screening colonoscopy with ambient lighting (75150 lux) may improve the differential change in ADR that occurs from the beginning to the end of the day. The use of ambient lighting was associated with a trend towards improved overall ADR after controlling for age, gender, and procedure time, which did not reach statistical significance. Improvement in ADR in later cases of the day may be due to improved symptoms of eye fatigue experienced by physicians performing endoscopy, although the present study does not prove that eye strain is the mechanism for this improved performance. Satisfaction by physicians and staff working in conditions of ambient lighting in our study was generally favorable, and resulted in a third of participating gastroenterologists choosing to adopt the routine use of ambient light. Future studies on optimal lighting settings for gastrointestinal endoscopy are warranted.

\section{Acknowledgements}

Funding was provided by a $\$ 2,500$ grant from the Advocate Charitable Foundation (\#2017-3).

\section{Competing interests}

The authors declare that they have no conflict of interest.

\section{Clinical trial}

clinicaltrials.gov

NCT04441242

TRIAL REGISTRATION: Non-Randomized, Single Center Clinical Trial NCT04441242 at clinicaltrials.gov

\section{References}

[1] Siegel RL, Miller KD, Fuchs HE et al. Cancer Statistics, 2021. CA Cancer J Clin 2021; 71: 7-33

[2] Siegel RL, Miller KD, Goding Sauer G et al. Colorectal cancer statistics, 2020. CA Cancer J Clin 2020; 70: 145-164

[3] Nishihara R, Wu K, Lochhead P et al. Long-term colorectal-cancer incidence and mortality after lower endoscopy. N Engl J Med 2013; 369: 1095-1105 
[4] Ran T, Cheng CY, Misselwitz B. Cost-Effectiveness of Colorectal Cancer Screening Strategies-A Systematic Review. Clin Gastroenterol Hepatol 2019; 17: 1969-1981.e15

[5] Rex DK, Boland CR, Dominitz JA et al. Colorectal Cancer Screening: Recommendations for Physicians and Patients from the U.S. MultiSociety Task Force on Colorectal Cancer. Am J Gastroenterol 2017; 112: $1016-1030$

[6] Kaminski MF, Wieszczy P, Rupinski M et al. Increased rate of adenoma detection associates with reduced risk of colorectal cancer and death. Gastroenterology 2017; 153: 98-105

[7] Almadi MA, Sewitch M, Barkun AN et al. Adenoma detection rates decline with increasing procedural hours in an endoscopist's workload. Can J Gastroenterol Hepatol 2015; 29: 304-308

[8] Lee CK, Cha JM, Kim WJ. Endoscopist fatigue may contribute to a decline in the effectiveness of screening colonoscopy. J Clin Gastroenterol 2015; 49: e51-e56

[9] Almario CV, Spiegel BMR. Does endoscopist fatigue impact adenoma detection rate? A review of the evidence to date Gastrointest Endosc 2017; 85: 611-613

[10] Teng TY, Khor SN, Kailasam M et al. Morning colonoscopies are associated with improved adenoma detection rates. Surg Endosc 2016; 30: $1796-1803$

[11] Wu J, Zhao SB, Wang SL et al. Comparison of efficacy of colonoscopy between the morning and afternoon: A systematic review and metaanalysis. Dig Liver Dis 2018; 50: 661-667

[12] Lee TJ, Rees C], Blanks RG et al. Colonoscopic factors associated with adenoma detection in a national colorectal cancer screening program. Endoscopy 2014; 46: 203-211

[13] Zorron Cheng Tao Pu L, Lu K, Ovenden A et al. Effect of time of day and specialty on polyp detection rates in Australia. J Gastroenterol Hepatol 2019; 34: 899-906

[14] Gurudu SR, Ratuapli SK, Leighton JA et al. Adenoma detection rate is not influenced by the timing of colonoscopy when performed in halfday blocks. Am J Gastroenterol 2011; 106: 1466-1471

[15] Lee A, Jensen CD, Marks AR et al. Endoscopist fatigue estimates and colonoscopic adenoma detection in a large community-based setting. Gastrointest Endosc 2017; 85: 601-610.e2

[16] Freedman JS, Harari DY, Bamji ND et al. The detection of premalignant colon polyps during colonoscopy is stable throughout the workday. Gastrointest Endosc 2011; 73: 1197-1206

[17] Vertinsky T, Forster B. Prevalence of eye strain among radiologists: influence of viewing variables on symptoms. AJR Am J Roentgenol 2005; 184: 681-686
[18] Krupinski EA, Berbaum KS, Caldwell RT et al. Long radiology workdays reduce detection and accommodation accuracy. J Am Coll Radiol 2010; 7: 698-704

[19] Porcar E, Pons AM, Lorente A. Visual and ocular effects from the use of flat-panel displays. Int J Ophthalmol 2016; 9: 881-885

[20] Artime Ríos EM, Lasheras FS, Suarez Sánchez A et al. Prediction of computer vision syndrome in health personnel by means of genetic algorithms and binary regression trees. Sensors (Basel) 2019; 19: 2800

[21] Schriewer T, Schulze R, Filippi A et al. The influence of ambient lighting on the detection of small contrast elements in digital dental radiographs. Clin Oral Investig 2013; 17: 1727-1731

[22] Hellén-Halme K, Petersson A, Warfvinge G et al. Effect of ambient light and monitor brightness and contrast settings on the detection of approximal caries in digital radiographs: an in vitro study. Dentomaxillofac Radiol 2008; 37: 380-384

[23] Hellén-Halme K, Lith A. Effect of ambient light level at the monitor surface on digital radiographic evaluation of approximal carious lesions: an in vitro study. Dentomaxillofac Radiol 2012; 41: 192-196

[24] Ohla H, Dagassan-Berndt D, Payer M et al. Role of ambient light in the detection of contrast elements in digital dental radiography. Oral Surg Oral Med Oral Pathol Oral Radiol 2018; 126: 439-443

[25] Pakkala T, Kuusela L, Ekholm M et al. Effect of varying displays and room illuminance on caries diagnostic accuracy in digital dental radiographs. Caries Res 2012; 46: 568-574

[26] Vertinsky T, Forster B. Prevalence of eye strain among radiologists: influence of viewing variables on symptoms. AJR Am J Roentgenol 2005; 184: 681-686

[27] Liukkonen E, Jartti A, Haapea M et al. Effect of display type and room illuminance in chest radiographs. Eur Radiol 2016; 26: 3171-3179

[28] Charwla AS, Ehsan S. Ambient illumination revisited: A new adaptation-based approach for optimizing medical imaging reading environments. Med Physics 2007; 34: 81-90

[29] Hayes JR, Sheedy JE, Stelmack JA et al. Computer use, symptoms, and quality of life. Optom Vis Sci 2007; 84: 738-744

[30] Active Physicians by Sex and Specialty. Physician Specialty Data Report 2018. AAMC; 2022: https://www.aamc.org/data-reports/ workforce/data/2018-physician-specialty-data-report-executivesummary

[31] Sheppard AL, Wolffsohn JS. Digital eye strain: prevalence, measurement and amelioration. BMJ Open Ophthalmol 2018; 3: e000146 